\title{
Pautas para la determinación efectiva del costeo basado en actividades en la industria bananera
}

\section{Guidelines for the effective determination of costing based on activities in the banana industry}

Mayra Lisseth Marfetán San Martín. ${ }^{1}$, Cecilia Ivonne Narváez Zurita. ${ }^{2}$, \& Juan Carlos Erazo Álvarez. ${ }^{3}$

\section{DOI: https://doi.org/10.33262/visionariodigital.v3i2.1.583}

\begin{abstract}
.
This article provides guidelines for the effective determination of costing based on activities in the banana industry; this sector is of vital importance in the Ecuadorian economy, so that this industry is recognized as one of the main economic activities that generate considerable income for the country, in this sense, the accounting information must be accurate and reliable, allowing evidence In addition, the costs of production are analyzed, and the cost drivers and the activities involved in obtaining this fruit are analyzed. In this investigation, the costs based on activities are determined, which are evident in the production processes, for the elaboration of $22 \mathrm{xu}$ conventional banana boxes, obtaining as a result real costs in each one of the elements of the cost; raw material, labor and indirect manufacturing costs in the Monte de Oro
\end{abstract} Keywords: Costs, Activities, Economy, Production

\section{Resumen}

El presente artículo proporciona pautas para la determinación efectiva del costeo basado en actividades en la industria bananera; este sector es de vital importancia en la económica ecuatoriana, de manera que, se reconoce a esta industria como una de las actividades económicas principales que generan ingresos considerables para el país, en este sentido, la información contable debe ser precisa y fidedigna, permitiendo evidenciar la realidad de los costos de producción, además, se analiza los

${ }^{1}$ Universidad Católica de Cuenca, Posgradista Maestría en Contabilidad y Auditoría, Cuenca, Ecuador, mmmarfetans431@psg.ucacue.edu.ec

2 Universidad Católica de Cuenca, Subdirección de Posgrado, Cuenca, Ecuador, inarvaez@ucacue.edu.ec

3 Universidad Católica de Cuenca, Subdirección de Posgrado, Cuenca, Ecuador, jcerazo@ucacue.edu.ec 
inductores de costeo y las actividades que intervienen para la obtención de esta fruta; en esta investigación se determinó efectivamente los costos basados en actividades, los cuales se evidencian en los procesos de producción, para la elaboración de cajas de banano convencional 22xu, obteniendo como resultado costos reales en cada uno de los elementos del costo; materia prima, mano de obra y costos indirectos de fabricación en la Finca Monte de Oro.

Palabras claves: costos $\mathrm{ABC}$, actividades, economía, producción

\section{Introducción.}

La exportación de banano representa el 2\% del PIB general y el 35\% del PIB del sector agrícola, debido a su importancia en la economía del Ecuador, el Gobierno ha establecido políticas a través de la Constitución del 2008 y acuerdos ministeriales en pro de mejorar las negociaciones, y lograr una adecuada distribución de los beneficios de este sector dentro de un negocio equitativo y comprometido social y ambientalmente, por estas razones se implementó un sistema de control liderado por el Aseguramiento de la Calidad del Agro, organismo que garantiza la calidad de los bananos de consumo y exportación (Ministerio de Comercio Exterior, 2017).

Las productoras de banano grandes, medianas o pequeñas, deben sujetarse a una buena administración, manteniendo una organización adecuada, además de apuntalar al establecimiento de metas y objetivos, con la finalidad de obtener resultados favorables mediante la optimización de recursos, permitiendo así, el crecimiento y consolidación del sector bananero. Los diferentes costos en los que incurren en este sector son muy variables, por lo que es necesario mantener un registro contable fidedigno de las transacciones mediante una planeación y organización adecuada a fin de obtener costos precisos, generando confianza en la toma de decisiones de los niveles jerárquicos respectivos.

En este sentido, George y Garner (2006) afirmana que, la contabilidad de costos emplea los principios contables, y su finalidad es proveer datos a los administradores y directivos de una entidad, instruyen a realizar cálculos que ayudan a descifrar el costo de originar los productos fabricados o de brindar servicios. Mientras que, Ramírez, García, y Pantoja (2010) mencionan que, la contabilidad de costos no es separada ni independiente; puesto que, al contrario, es parte integral del sistema contable de una organización, en tal sentido, se la considera como un subsistema de la misma, en la cual se registran operaciones correspondientes a los valores monetarios que se originan o se determinan en la elaboración de los productos.

Wayne y Ledesma (2015) consideran que, la contabilidad de costos tiene una orientación gerencial por lo que, proporciona información fundamental a los administradores de empresas a través de una adecuada planificación, esta contaduría ayuda a los altos mandos a tomar mejores decisiones dentro de una organización, es decir, proporciona información indispensable para la entidad; la teoría de costos tiene una relación estrecha con la contabilidad general, encargándose del análisis, registros y controles de los valores económicos que incurren o invierten en la adquisición o elaboración de los 
productos en una organización empresarial. Por su parte, Lambreton y Garza (2016) aseguran que , la contabilidad de costos se encarga de suministrar informacion tanto a la contabilidad financiera como a la contabilidad administrativa, aportando al cumplimiento de los principales objetivos contables para el crecimiento de la entidad.

Los elementos del costo para la elaboración de un producto o la prestación de un servicio, según, Fabriozzi, Kole, y Adelberg (1997), Cuevas (2010) y Cano (2013), son los siguientes:

(a) Materiales directos: son las materias primas que incurren directamente en la fabricación y que forman parte integral de un bien, las cuales deben ser: identificables directamente en el bien, el valor es significativo y su uso es relevante dentro del producto.

(b) Mano de obra directa: constituyen aquellos costos laborales del trabajo humano que no se ven físicamente reflejados como: los sueldos y aportes patronales, que incurren directamente en la elaboración del producto.

(c) Costos indirectos de fabricación (C.I.F): son aquellos que pueden sustituir, a éstos también se los conoce como gastos generales de fabricación, tales como; materiales indirectos, mano de obra indirecta y costos generales de fábrica.

Una vez definidos los elementos del costo se determina el costo de un producto, utilizando un estado de costos que demuestra en unidades monetarias lo que realmente se utilizó a lo largo de este proceso productivo, con la ayuda de esta herramienta se evidencian los costos reales. En la tabla 1 se muestra el formato del estado de costos:

Tabla 1. Formato del estado de costos

\begin{tabular}{|c|c|}
\hline Detalle & Valores \\
\hline Materiales Directos & $\operatorname{xxxx}$ \\
\hline Inventario final de Materiales Directos & $\operatorname{xxxx}$ \\
\hline$=$ Costo de los materiales directos & $\operatorname{xxxx}$ \\
\hline + Mano de obra & $\operatorname{xxxx}$ \\
\hline$+\mathrm{CIF}$ & $\operatorname{xxxx}$ \\
\hline$=$ Costos de producción del presente periodo & $\operatorname{xxxx}$ \\
\hline$=$ Costo total de productos en proceso & $\operatorname{xxxx}$ \\
\hline (-) Inventario Final de productos en proceso & $\operatorname{xxxx}$ \\
\hline$=$ Costo de productos terminados en el presente periodo & $\mathrm{XXXX}$ \\
\hline
\end{tabular}

Fuente: Cano (2013) 


\section{Sistemas de costeo}

\section{a) Costos por órdenes de trabajo.}

Horngren, Datar y Rajan (2012) determinan que, los costos por ordenes de producción se dan en una unidad o en varias unidades de productos y servicios utilizando distintos recursos para procesar cada orden de trabajo;el bien o el servicio es casi siempre en una sola unidad y se acopian los costos de manera apartada para cada producto o servicio. Cano (2013) afirma que, también se lo llama por lotes o entregas específicas, utilizada en empresas de producción estandarizadas, o que diseñan un producto acorde a las necesidades del cliente, además señala que en este se considera los materiales directos y mano de obra directa como reales, mientras que los CIF son presupuestados.

Los costos por órdenes de trabajo son utilizados por empresas productivas que se dedican a la fabricación de bienes o servicios con especificaciones por parte de cliente, procesos que no son continuos, utilizan el ensamble o por lotes, en donde las unidades son perfectamente identificables en el proceso de producción. En este sistema no se establece periodos de tiempo para la consecución de la obra, puesto que en algunos casos las etapas son cortas mientras que en otras puede alargarse a varios meses.

\section{b) Costos por procesos.}

El sistema de costos por procesos registra controla y acumula los costos por departamentos o centro de costos. Polimeni, Fabriozzi, Kole y Adelberg (1997) indican que, este sistema es utilizado en empresas industriales y productivas que fabrican productos en los que no se considera gustos y preferencias de los clientes; se aplica cuando los productos se manufacturan mediante técnicas de producción masivas o procesamiento continúo. Además, este sistema consta de informes y flujos.

El informe de costos de producción lo hace cada departamento y contiene 4 secciones:

$\checkmark \quad$ Cantidad: unidades que entran y salen de los materiales de un departamento a otro del proceso productivo.

$\checkmark \quad$ Producción equivalente: es aquella que registra y controla las unidades terminadas más aquellas unidades que están en proceso y se las tiene que considerar como terminadas, en razón que el costo es estimado.

$\checkmark \quad$ Costos para contabilizar: registra y controla los costos unitarios que se generan en cada uno de los departamentos a partir de la producción equivalente.

$\checkmark$ Costos contabilizados: registra y controla los costos totales de cada uno de los departamentos, centros de costos. 
Respecto a los tipos de flujo se puede diferenciar los siguientes:

$\checkmark \quad$ Secuencial: representa la continuidad de la materia prima.

$\checkmark \quad$ Paralelo: hay varios departamentos y la materia prima entra en diferentes partes.

$\checkmark \quad$ Selectivo: parte de una materia prima común de la cual se derivan diferentes tipos de productos y subproductos; subproductos- directamente a la venta.

Horngren, Datar y Rajan (2012) afirman que, las asociaciones que manejan este procedimiento son aquellas que producen productos semejantes o afines de un bien o servicio, la valoración de los períodos de culminación de las unidades y la concesión de los costos a las unidades. Mientras que, Ramírez, García, y Pantoja (2010) aseveran que, los costos por ordenes de producción se realizan mediante pedidos $u$ órdenes de trabajo de manera concreta, denominándolo también como un sistema de costos por lotes.

\section{Métodos de Costeo.}

\section{a) Método de coste total.}

Backer (como lo citó Morillo, 2005) establece que, el método de costeo total acopia todos los costes de la fabricación, tanto costos afirmados como variables, éstos son estimados como parte del importe de las mercancías elaboradas, bajo la premisa de que todos son necesarios para transformar un producto. Cano (2013) menciona que, este método de costeo se lo conoce como absorbente siendo el más habitual puesto que considera el total de los costos de la producción en todas sus fases, con los productos principales y secundarios empleados como son los costos fijos y variables. Este sistema de costeo total muestra restricciones cuando se maneja la intención de valuar y tomar decisiones, no obstante, esta restrictiva no quiere decir que dicho sistema sea erróneo o inadecuado.

\section{b) Método de costeo variable.}

Baldini (2008) establece que, este método carga los costos según los elementos sean determinados, considera si son proporcionales con el volumen de producción o de ventas. En cambio, Cano (2013) señala que, a este método se lo conoce como costeo marginal o directo, ya que los costos varian en correspondencia con la producción y ventas, por ello, se conisderan el valor de los materiales, mano de obra y de los costos indirectos de fabricación variables.

\section{c) Método de costeo basado en actividad ABC}

Sáez (como lo cito Morillo, 2005) menciona que, este método parte de la diferencia entre costos inmediatos y costos indirectos, concerniendo los últimos con las operaciones que 
realiza la entidad. Los movimientos se planean de tal manera que los costos indirectos surgen como directos a las diligencias, desde donde se les aplica a los bienes (objeto de costos), según la cantidad de actividades consumidas por cada objeto de costos. De manera que, el costo finito está consentido por los costos directos y por los costos asociados a ciertas actividades, estimadas como las que aumentan valor a los bienes.

El sistema de costos basado en actividades consiste en identificar cuáles son las actividades de mayor importancia en la producción de un bien o un servicio, por lo que su implementación permite obtener costos exactos para la entidad, ofreciendo información necesaria para la correcta toma de decisiones (Ramirez , 2008), al respecto, Horngren, Datar, y Rajan (2012) señalan que este sistema se caracteriza por establecer los objetos del costos que son funadamentales para identificar las actividades de todas las ocupaciones de valor que intervinen en un proceso de producción.

Laporta (2016) plantea que, los costos basados en actividades, son una alternativa al tratamiento de los CIF, expone que no son los bienes, los departamentos, ni los servicios los que ejecutan los costos, sino esencialmente las actividades. Los conceptos propuestos en base a los autores se basan en un mismo criterio en los costos basados en actividades, pero el concepto de Laporta (2016) es el más exacto por que evidencia un estudio más analítico, que brinda un grado información satisfactoria para un correcto análisis.

\section{Estructuración del sistema de costos en base actividades ABC}

Ortíz y Rivero (2006) establecen que se deben considerar los siguientes parámetros para elaborar una estructura de costos basados en actividades:

$\checkmark$ Analizar las actividades de acuerdo a la necesidad que la entidad requiera.

$\checkmark$ Definir los centros de costo y examinar y/o acoger un plan de cuentas.

$\checkmark$ Instaurar y/o ratificar los supuestos por centro de costo que deriva a la realización de actividades.

$\checkmark$ Consolidar la información contable y obtener reportes.

Además, el autor recalca que, en una ejecución de programaciones y sistemas contables tomados parcialmente, las estructuras de costos se verán limitadas y no será de ayuda a la entidad.

Pérez (2014) plantea que, la estructura de costos es el ADN de las organizaciones, por lo que es preciso dedicar tiempo para examinar evidencias tales como el objeto de la empresa, reconocer el fin de los costos, diagnosticar los elementos que lo abarcan, los paradigmas de inventarios y demás aspectos, que si no son correctamente analizaos pueden conllevar a la aplicación de una estructura de costos que no calcule, pondere y evalué al sujeto u organización. 


\section{Objeto del costo}

El objeto de costo es la razón por la cual se genera una actividad, estos contienen contratos, clientes, servicios, proyectos y productos, que pueden ser clasificados en costos directos e indirectos (Vanden, 2007).

$\checkmark$ Costos Directos: Son todos aquellos costos de los cuales se puede realizar un seguimiento de los recursos factibles empelados, hacia el objeto de costos. En este sentido, los que física y económicamente se visualizan en algún trabajo o centro de costos.

$\checkmark$ Costos Indirectos: Estos hacen referencia a los costos de los cuales no se puede realizar un seguimiento hacia el objeto de costos, de forma económicamente factible.

\section{Cadena de Valor}

Heredia (2008) asegura que, cadena de valor son las activuades que agregan valor en la elaboración de un producto, desde la compra de los insumos hasta la creación de un producto o servicio. Se aprecia ese plus en precios del producto o servicio. Es el total del costo de las actividades para diseñar, elaborar, mercadear, entregar y apoyar el bien o servicio. Por su parte, Vergíu (2013) afirma que, la cadena de valor es una herramienta y un modelo teórico que permite describir el desarrollo de las actividades de una entidad empresarial para generar valor al cliente final, las actividades de valor se dividen en dos grandes grupos: primarias y secundarias. Las primarias son las que intervienen en la creación física del producto, en su venta, y transferencia al cliente, así como en la asistencia o servicio posterior a la venta, mientras que, las actividades secundarias respaldan a las primarias y viceversa, al ofrecer materias primas, tecnología, recursos humanos y diversas funciones generales.

Ortega (2016) establece que, las actividades se igualan en conjuntos que forman el total de los procesos productivos de una organización, siendo estos ordenados de forma secuencial y paralela, obteniendo como resultado diferentes estados de costo que se almacenan en la producción y el valor que agregan a cada proceso, al momento de utilizar este sistema las empresas realizan tareas que crean valor en los productos y rechazan aquellos que no aportan valor en los factores productivos.

\section{Beneficios del sistema de costos basado en actividades ABC}

El sistema de costeo $\mathrm{ABC}$, genera importantes beneficios a las organizaciones que lo emplean, entre otros, se destacan los siguientes:

$\checkmark$ Permite entender el comportamiento de los costos de la entidad y admite hacer presupuestos financieros, mediante la información que se obtiene a favor del incremento o disminución en los niveles de actividad. 
$\checkmark$ El sistema de costeo ABC suministra información sobre las causas que generan la actividad y el análisis de cómo se realizan las tareas.

$\checkmark$ Permite tener un enfoque real y de forma horizontal de lo que ocurre en la empresa.

$\checkmark$ El sistema ABC facilita cierta información que disminuye los costos de estudios especiales que algunos centros departamentales realizan para perfeccionar al sistema de costos tradicional.

Si se aumenta el nivel de información y además se disminuye los costos del propio centro de costos, el sistema de costeo $\mathrm{ABC}$ resulta sencillo y real, en cuanto estos se basan en hechos reales y es completamente objetivo, por lo que, se encuentra sustentado en las actividades y, por tanto, es complejo que pueda ser maniobrado de alguna manera, ya que los datos obtenidos son reales. El sistema de costeo $\mathrm{ABC}$ ha sido probado y certificado por varios autores, así como aplicado en todo tipo de industrias, por lo que se torna viable para su aplicación en cualquier circunstancia (Plaza, 2013).

\section{Inductores del costo}

Heredia (2008) asegura que, los iductores del costos se establecen a través de múltiples tasas aplicadas en cada tarea, actividad, proceso, unidad o lote, de tal manera que los centros de acumulación de costos se encuentren asociados en las actividades y valorados de acuerdo a la asignación establecida por la entidad empresarial. Barraza (2013) menciona que los drivers o inductores del costo sirven como conexión entre las actividades que la entidad realiza en la elaboración de un producto y sus costos indirectos de fabricación respectivos y que se pueden relacionar también con el producto terminado donde cada indicador de actividad debe estar definido en unidades que seas perfectamente identificables.

La principal característica en el sistema de costos basado en actividades es la medición, su relación no se basa directamente con el volumen del producto, ya que, se analiza la conducta de la actividad y de los costos que recaen sobre ella, en función de indicadores no relacionados con los mismos. Las etapas que se deben seguir para determinar el costo de un servicio o producto a través de este sistema basado en actividades son:

$\checkmark \quad$ Los costos deben ser identificados como directos, tales como la materia prima se imputan a los productos. Los costos indirectos se reparten por centros de responsabilidad, estos a su vez se pueden dividir entre las actividades, en esta etapa se reagruparían los costos en relación con las actividades principales y auxiliares.

$\checkmark \quad$ Los costos de las actividades auxiliares se asignan a las actividades principales, en esta última etapa, se asignarían los costos de las actividades principales a los productos, a través de los inductores de costos. 


\section{Actividades}

Heredia (2008) establece que, las actividades se agrupan en cuatro categorías de acuerdo a su proceso productivo:

$\checkmark$ Centros de actividades de servicios: Las actividades comerciales dentro del negocio, los servicios prestados por estos centros que son generalmente ejecutados para otros centros de costos.

$\checkmark$ Centros de actividades operacionales de apoyo: En este centro de costo, las actividades soportan las actividades de operaciones directas.

$\checkmark$ Centros de actividades administrativas: Son las actividades referentes a la administración y dirección.

$\checkmark$ Centro de actividades operativas: En este centro de costeo las actividades se procesan directamente los bienes y servicios ofrecidos por la empresa o los departamentos directos.

Barraza (2013) afirma que las actividades son tareas que ejecutan las personas para la elaboración de un bien o servicio. Las actividades en el campo industrial se relacionan en conjuntos o grupos que forman el total de procesos productivos, los cuales son ordenados de forma lógica, secuencial y simultánea para la elaboración de productos con valor agregado que le adicionan en cada proceso. Finalmente, la realización de actividades consume recursos de la empresa que se acumulan por medio de los costos. Por su parte, Golpe (2017) considera que, con la implementación de un sistema de costeo $\mathrm{ABC}$ se logra determinar las actividades que incurren en la elaboración de un bien o un servicio, lo cual permite mejorar los procesos y atender las necesidades de la empresa y de los clientes. En este sentido, las actividades se clasifican bajo los siguientes parámetros:

$\checkmark$ Por funciones: compras, producción, ventas.

$\checkmark$ Por su naturaleza: fundamentales o discrecionales.

$\checkmark$ Por su relación con el objetivo de costo: por volumen de producción, relacionadas con los Procesos productivos, de sostenimiento o de infraestructura.

$\checkmark \quad$ Por su aportación a la generación de valor: con valor añadido o sin valor añadido.

Por su parte, Cano (2013) señala que las activiades son un conjunto de tareas elaboradas por personas o maquinas con cualidades específicas de la organización, las mismas que, se agrupan en tareas homogéneas susceptibles de cuantificar tal como se puede apreciar en la tabla 2. 
Tabla 2. Clasificación de actividades.

Actividades

Actividades de nivel unitario

Actividades nivel lote

Actividades a nivel línea

Actividades a nivel línea

\section{Descripción}

Cuando se elabora una entidad de un bien y el consumo de recursos depende de las unidades producidas.

Elaboración de varias unidades en el mimo tiempo.

Elaboración de productos de diferentes gamas.

Son operaciones que se desarrollan dentro de la empresa como operaciones administrativas contables, financieras las primarias que se contabilizan como costo y las otras operaciones tales como asesoría fiscal, laboral, jurídica consideradas con secundarias se contabilizarían como gasto.

\section{Metodología.}

La presente investigación se contextualizó bajo el enfoque mixto, los datos obtenidos fueron datos cuantitativos y cualitativos mediante la utilización de encuestas y entrevistas en relación a los costos en base a actividades; el estudio tuvo un alcance descriptivo-explicativo DEXPLIS, en lo que respecta a la finalidad de la investigación esta fue transversal, dado que, la recopilación de información se la realizó en un tiempo específico.

Se estableció el uso del método histórico- lógico en la búsqueda de diferentes conceptos sobre los costos en base a actividades, siguiendo un orden lógico para su desarrollo; el método inductivo- deductivo se aplicó a partir del análisis de los conceptos generales sobre la contabilidad de costos llegando a la particularidad de cada componente; el método estadístico se aplicó en el procesamiento de la información obtenida mediante instrumentos empleados en la investigación; el método analítico-sintético se empleó para la descomposición de las variables descifrando todos sus componentes llegando a un concepto general.

En esta investigación el universo de estudio estuvo conformado por 17 personas, que trabajan en las áreas contable, administrativa y de producción en los niveles estratégico, táctico y operativo, en la Finca Monte de Oro, ubicada en el cantón El Guabo. 
Tabla 3. Población

Nivel Estratégico

\begin{tabular}{llr}
\hline Área & Cargo & No. Personas \\
\hline Gerencia & Propietario & 1 \\
Administración & Administrador & 4 \\
Nivel Estratégico & & \\
Contabilidad & Contador & 1 \\
Contabilidad & Auxiliar contable & 8 \\
Nivel Operativo & & 1 \\
Campo & Agrónomo & 1 \\
Campo & Mayordomo & 1 \\
Campo & Fumigador & 17 \\
& Total & \\
\hline
\end{tabular}

\section{Resultados.}

Los resultados obtenidos mediante la aplicación de los instrumentos en la Finca Monte de Oro se mencionan a continuación:

\section{$\checkmark$ Estructura de costos}

La empresa no cuenta con una estructura de costos que facilite el cumplimiento de los procesos de producción en cada una de sus etapas, lo que ocasiona que no se obtengan información adecuada de sus costos.

Los costos de producción de la finca no han cambiado aun cuando se implementado mejoras en la producción de banano.

\section{$\checkmark$ Elementos del Costo}

El $100 \%$ de los encuestados desconocen los elementos del costo y su clasificación, por ende, existe una deficiente determinación de los costos afectando el margen de ganancia de la Finca Monte de Oro.

\section{$\checkmark$ Costos en base a actividades}

El $100 \%$ de los encuestados afirman que los costos de producción no se agrupan por actividades para la obtención de cajas de banano convencional 22xu, lo que evidencia que actualmente se está empleando un sistema tradicional que no permite establecer los costos reales.

Las deficiencias identificadas en la unidad de análisis permiten inferir que la aplicación del sistema de costeo tradicional que se emplea, no proporciona un escenario claro del comportamiento de los costos y del margen de utilidad cuando existe una variación en más o 
en menos del volumen de actividades, por lo que, la información disponible no resulta de gran utilidad a la hora de decidir en temas relacionados a las ventas o producción. Ante este escenario, y con la finalidad de contar con una herramienta que permita rastrear los costos a partir de la relación causa - efecto entre uno de los factores de producción y una actividad, se propone pautas para un coste efectivo a través del sistema $\mathrm{ABC}$.

\section{Propuesta}

La Finca Monte de Oro cuenta con 17 lotes de 5 hectáreas cada uno, por ende, los cálculos presentados en esta investigación se realizaron por lote.

Figura 1. Esquema de la propuesta de costos en base a actividades

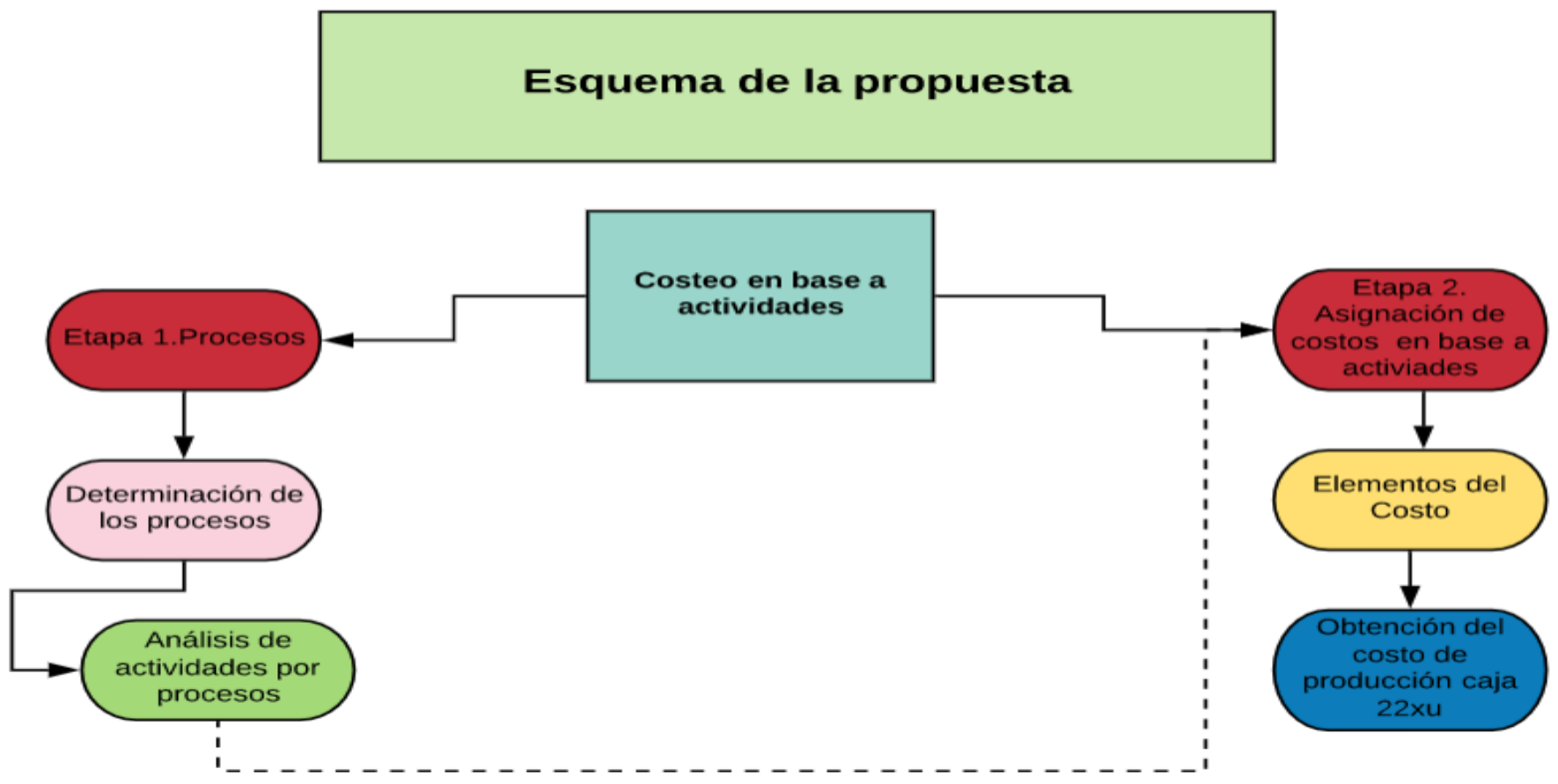

\section{Determinación de los procesos}

La determinación de los procesos como parte de la primera etapa del esquema de la propuesta de la estructura de costos en base a actividades se detalla a continuación: 
Figura 2. Determinación de los procesos de la Finca Monte de Oro

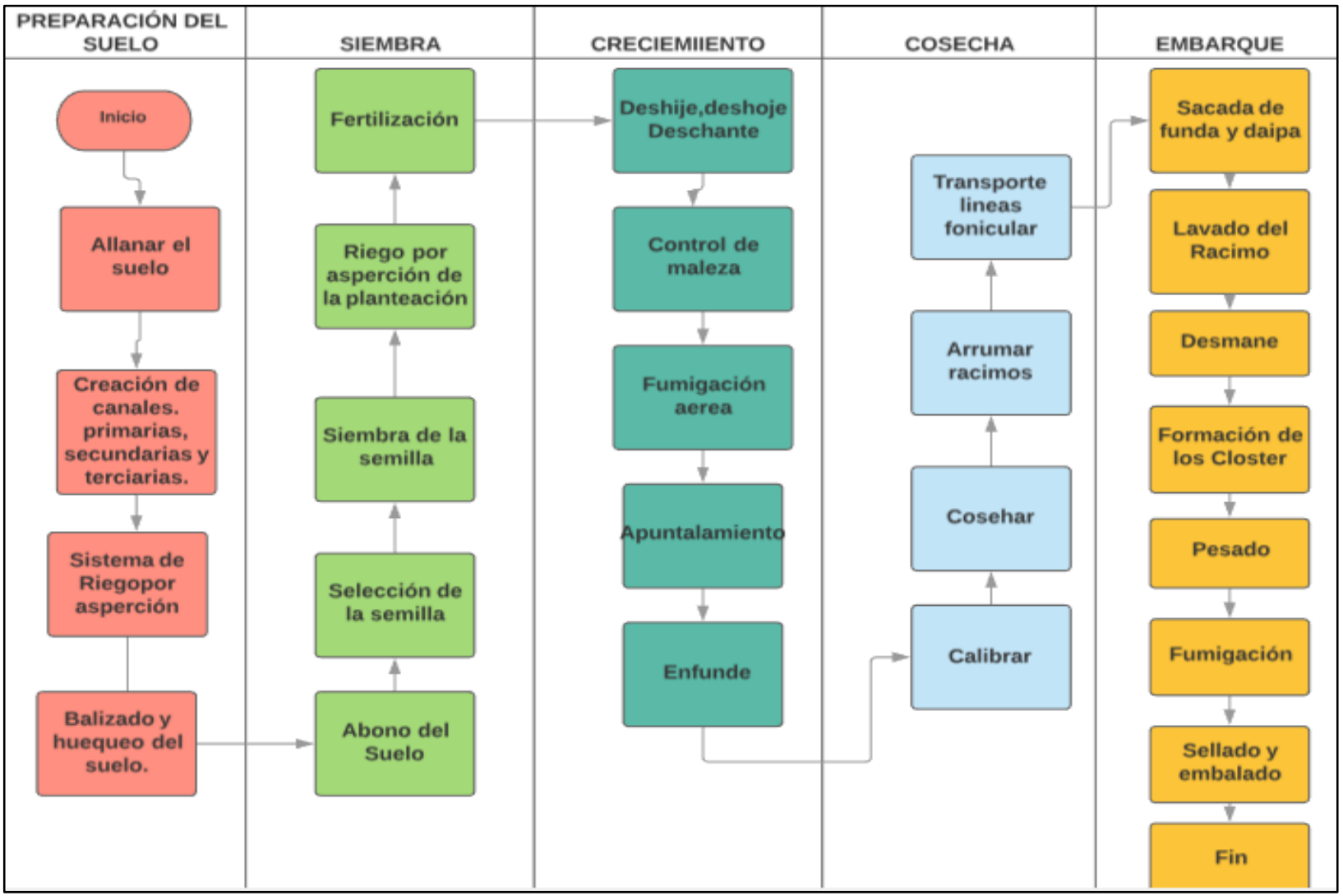

\section{Análisis de las actividades por procesos.}

a) Preparación del suelo: Para la preparación del suelo primero se debe elaborar el arado del suelo, después se deben crear los canales donde se encuentran divididos en canales primarios, secundarios y terciarios, luego que estos canales estén listos se procede a implementar el sistema de riego por aspersión.

b) Siembra: Para la siembra del banano se baliza el suelo, se realizan orificios donde se procede a desinfectar con nematicidas y abonar el suelo. En esta etapa se selecciona la semilla si son colines o hijos de la planta principal.

c) Crecimiento: Para el crecimiento de la planta y de la fruta de banano se realiza el riego de 2 a 3 veces por semana, se fertiliza cada semana de acuerdo a un programa de fertilización que se lo hace semestralmente, se realiza el deshije, deshoje y deschante, control de la maleza, fumigación aérea para fitosanitaria de sigatoca negra, apuntalamiento con puntal o suncho, se procede a enfundar la fruta eliminando bacterias y laterales, se incorpora la protección ya sea de disco o daypa. 
d) Cosecha: Para la cosecha del banano se calibra la fruta, se cosecha de la planta y se procede a arrumar los racimos para luego transportarlos por el camino funicular por todo el campo, en cada trayecto de traspaso a la empacadora van 20 racimos colgados en garruchas del embarque.

e) Embarque: Una vez el racimo de banano llega a la empacadora se procede a sacar la protección la daypa o el disco, se lava el racimo, se desmana el racimo, se forman los closter, se procede a pesar y fumigar la fruta, se realiza el embalado y sellado, posteriormente se traslada a los conteiner que irán al puerto de entrega.

\section{Elementos del costo}

\section{a) Materia prima}

La materia prima son los colines.

Tabla 4. Materia prima

\begin{tabular}{ccc}
\hline & Valor & Valor \\
Colines & Unitario & Total \\
\hline
\end{tabular}

b) Mano de obra

$\begin{array}{lll}6500 & 0.77 \quad 5005.00\end{array}$

Tabla 5. Preparación del suelo y siembra

\begin{tabular}{ccccccc}
\hline No. & Empleados & Labores & $\begin{array}{c}\text { Horas } \\
\text { mensuales }\end{array}$ & $\begin{array}{c}\text { Sueldo } \\
\text { normal }\end{array}$ & $\begin{array}{c}\text { Horas } \\
\text { trabajadas }\end{array}$ & $\begin{array}{c}\text { Sueldo por } \\
\text { hora } \\
\text { trabajada }\end{array}$ \\
\hline $\mathbf{1}$ & Empleado1 & Varias & 36 & 90,92 & 1.15 & 41.40 \\
$\mathbf{2}$ & Empleado2 & Varias & 40 & 90,92 & 1.15 & 46.00 \\
$\mathbf{3}$ & Empleado3 & Varias & 40 & 90,92 & 1.15 & 46.00 \\
\hline & & Total & & 454,60 & & 133.40 \\
\hline
\end{tabular}

\begin{tabular}{ccc}
$\begin{array}{c}\text { Valor } \\
\text { hora }\end{array}$ & Ingresos por horas trabajadas & 133.40 \\
hombre & $=\begin{array}{c}\text { Ingresos Mensuales + Provisiones } \\
\text { Mensuales }\end{array}$ & \multicolumn{2}{c}{454.60}
\end{tabular}


Tabla 6. Crecimiento y mantenimiento

\begin{tabular}{|c|c|c|c|c|c|c|c|c|}
\hline No. & Empleados & Labores & $\begin{array}{c}\text { Horas } \\
\text { mensuales }\end{array}$ & $\begin{array}{c}\begin{array}{c}\text { Sueldo } \\
\text { seis } \\
\text { semanas }\end{array} \\
\end{array}$ & $\begin{array}{c}\text { Horas } \\
\text { trabajadas }\end{array}$ & $\begin{array}{l}\text { Sueldo por hora } \\
\text { trabajada }\end{array}$ & XIII & XIV \\
\hline 1 & Empleado 1 & Mantenimiento & 240.00 & 272.77 & 1,14 & 272.77 & 45.46 & 45.46 \\
\hline 2 & Empleado 2 & Enfundadores & 240.00 & 272.77 & 1,14 & 272.77 & 45.46 & 45.46 \\
\hline 3 & Empleado 3 & Mayordomo & 240.00 & 272.77 & 1,21 & 290.76 & 48,46 & 45.46 \\
\hline 4 & Empleado 4 & Varios & 240.00 & 272.77 & 1,14 & 136.39 & 45.46 & 45.46 \\
\hline 5 & Empleado 5 & Varios & 240.00 & 272.77 & 1,14 & 136.39 & 45.46 & 45.46 \\
\hline \multirow[t]{2}{*}{6} & Empleado 6 & Bodeguero & 240.00 & 272.77 & 1,14 & 136.39 & 45.46 & 45.46 \\
\hline & & & Total $=$ & $1,636.62$ & - & 954.71 & 227.30 & 272.76 \\
\hline
\end{tabular}

Tabla 7. Cosecha y Embarque

\begin{tabular}{rllrrrr}
\hline No. & Empleados & Labores & $\begin{array}{c}\text { Horas } \\
\text { mensuales }\end{array}$ & $\begin{array}{c}\text { Sueldo seis } \\
\text { semanas }\end{array}$ & $\begin{array}{c}\text { Horas } \\
\text { trabajadas }\end{array}$ & $\begin{array}{c}\text { Sueldo por } \\
\text { hora } \\
\text { trabajada }\end{array}$ \\
\hline $\mathbf{1}$ & Empleado 1 & Cuadrilla & 48.00 & 84.00 & 1.00 & 48.00 \\
$\mathbf{2}$ & Empleado 2 & Cuadrilla & 48.00 & 84.00 & 1.00 & 48.00 \\
$\mathbf{3}$ & Empleado 3 & Cuadrilla & 48.00 & 84.00 & 1.00 & 48.00 \\
$\mathbf{4}$ & Empleado 4 & Empleado & 48.00 & 84.00 & 1.00 & 48.00 \\
$\mathbf{5}$ & Empleado 5 & Empleado & 48.00 & 84.00 & 1.00 & 48.00 \\
$\mathbf{6}$ & Empleado 6 & Empleado & 48.00 & 84.00 & 1.00 & 48.00 \\
$\mathbf{7}$ & Empleado 7 & Empleado & 48.00 & 84.00 & 1.00 & 48.00 \\
\hline
\end{tabular}

\begin{tabular}{|c|c|c|}
\hline \multirow{2}{*}{$\begin{array}{c}\text { Valor } \\
\text { hora } \\
\text { hombre = }\end{array}$} & Ingresos por horas trabajadas & 336.00 \\
\hline & Ingresos Mensuales + Provisione & \\
\hline
\end{tabular}


Tabla 8 Costo Mano de Obra por actividad

Descripción

Valor

Actividad Preparación del suelo y

siembra

0,29

Actividad crecimiento

0.44

Actividad cosecha y embarque

0.57

Valor hora hombre

1.30

\section{c) Costos indirectos de fabricación}

Tabla 9. Costos Indirectos de Fabricación que incurren en la bananera en lote de 5 has.

\begin{tabular}{|c|c|c|c|c|c|}
\hline Materiales & Cantidad & $\begin{array}{c}\text { Unidad } \\
\text { de } \\
\text { medida }\end{array}$ & $\begin{array}{l}\text { Costo unitario } \\
\text { del producto }\end{array}$ & $\begin{array}{c}\text { Costo total } \\
\text { por Has. }\end{array}$ & Inductor del costo \\
\hline Cinta & 4.00 & Rollos & 2.79 & 11.16 & No Orden de pedido \\
\hline Pañuelos & 600.00 & Unidades & 0.05 & 30.00 & No Orden de pedido \\
\hline Fundas & 300.00 & Unidades & 0.08 & 24.00 & No Orden de pedido \\
\hline Daypas & 432.00 & Unidades & 0.06 & 25.23 & No Orden de pedido \\
\hline Gomas & 1.40 & Unidad & 21.68 & 30.35 & No Orden de pedido \\
\hline Cloro & 1.36 & Libras & 1.21 & 1.65 & No Orden de pedido \\
\hline Removedor & 1.00 & Litro & 1.20 & 1.20 & No Orden de pedido \\
\hline Piola & 1.00 & Rollo & 1.70 & 1.70 & No Orden de pedido \\
\hline Lustre & 2.00 & Fundas & 0.30 & 0.60 & No Orden de pedido \\
\hline Protectores & 3.00 & Unidades & 0.04 & 0.12 & No Orden de pedido \\
\hline Cartones & 231.00 & Unidades & 0.47 & 108.57 & No Orden de pedido \\
\hline Etiqueta & 1.00 & Rollo & 1.90 & 1.90 & No Orden de pedido \\
\hline \multicolumn{2}{|c|}{ Total Materiales } & & 32.48 & 236.48 & \\
\hline Herramientas & Cantidad & $\begin{array}{c}\text { Unidad } \\
\text { de } \\
\text { medida }\end{array}$ & $\begin{array}{l}\text { Costo unitario } \\
\text { del producto }\end{array}$ & $\begin{array}{c}\text { Costo total } \\
\text { por Has. }\end{array}$ & Inductor del costo \\
\hline Garruchas & 2.00 & Convoy & 0.06 & 0.12 & No Orden de pedido \\
\hline Podones & 6.00 & Unidades & 1.15 & 6.9 & No Orden de pedido \\
\hline
\end{tabular}




\begin{tabular}{|c|c|c|c|c|c|}
\hline Cucharetas & 5.00 & Unidades & 1.50 & 7.5 & No Orden de pedido \\
\hline Cunas & 8.00 & Unidades & 0.17 & 1.36 & No Orden de pedido \\
\hline Platos & 2.00 & Unidades & 0.21 & 0.42 & No Orden de pedido \\
\hline Rechazadora & 4.00 & Unidades & 0.15 & 0.6 & No Orden de pedido \\
\hline \multicolumn{3}{|c|}{ Total Herramientas } & \multicolumn{2}{|r|}{16.9} & \\
\hline Fumigación & Cantidad & $\begin{array}{l}\text { Unidad } \\
\text { de } \\
\text { medida }\end{array}$ & $\begin{array}{l}\text { Costo unitario } \\
\text { del producto }\end{array}$ & $\begin{array}{l}\text { Costo total } \\
\text { por Has. }\end{array}$ & Inductor del costo \\
\hline Echo & 8.75 & Litro & 7.55 & 66.06 & Orden por Lotes \\
\hline $\begin{array}{l}\text { Coadyuvante x-50 } \\
\text { Servicio de } \\
\text { fumigación }\end{array}$ & 1 & Litro & 22.22 & 22.22 & $\begin{array}{l}\text { Orden por Lotes } \\
\text { Orden por Lotes }\end{array}$ \\
\hline Emthane & 7.5 & Litro & 6.9 & 51.75 & Orden por Lotes \\
\hline $\begin{array}{l}\text { Coadyuvante x-50 } \\
\text { Servicio de } \\
\text { fumigación }\end{array}$ & 5 & $\begin{array}{l}\text { Litro } \\
\text { Litro }\end{array}$ & 22.22 & 54.50 & $\begin{array}{l}\text { Orden por Lotes } \\
\text { Orden por Lotes }\end{array}$ \\
\hline Siganex & 2.5 & Litro & 22.25 & 55.63 & Orden por Lotes \\
\hline Impulse & 2 & Litro & 34.9 & 69.80 & Orden por Lotes \\
\hline $\begin{array}{l}\text { Coadyuvante x-50 } \\
\text { Aceite agrícola } \\
\text { importado } \\
\text { Servicio de } \\
\text { fumigación }\end{array}$ & 0.5 & $\begin{array}{l}\text { Litro } \\
\text { Litro }\end{array}$ & 22.22 & 11.11 & $\begin{array}{l}\text { Orden por Lotes } \\
\text { Orden por Lotes }\end{array}$ \\
\hline Echo & 8.75 & Litro & 7.55 & 66.06 & Orden por Lotes \\
\hline Coadyuvante $x-50$ & 1 & Litro & 22.22 & 22.22 & Orden por Lotes \\
\hline $\begin{array}{l}\text { Servicio de } \\
\text { fumigación }\end{array}$ & 5 & & 10.9 & 54.50 & Orden por Lotes \\
\hline
\end{tabular}

Total de fumigación

\begin{tabular}{lrrrrr}
\hline & Cantidad & $\begin{array}{c}\text { Unidad } \\
\text { de } \\
\text { medida }\end{array}$ & $\begin{array}{c}\text { Costo unitario } \\
\text { del producto } \\
\text { por gramo }\end{array}$ & $\begin{array}{c}\text { Costo total } \\
\text { por Has. }\end{array}$ & Inductor del costo \\
\hline Nitrogreen & 75000 & Gramos & 0.0004754 & 35.66 & Orden por Lotes \\
Sulfati de Potasio & 25000 Gramos & 0.0006632 & 16.58 & Orden por Lotes \\
Muriato de Potasio & 50000 Gramos & 0.000008 & 0.40 & Orden por Lotes
\end{tabular}




\begin{tabular}{|c|c|c|c|c|c|}
\hline Kieserete & 25000 & Gramos & 0.0002746 & 6.87 & Orden por Lotes \\
\hline Sulfato de Zinc & 15000 & Gramos & 0.0014364 & 21.55 & Orden por Lotes \\
\hline Naturcomplet G & 12500 & Gramos & 0.00153 & 19.13 & Orden por Lotes \\
\hline Bazemax & 150000 & Gramos & 0.0003592 & 53.88 & Orden por Lotes \\
\hline \multicolumn{3}{|c|}{ Total Fertilizantes } & 0.0047468 & \multicolumn{2}{|l|}{154.05} \\
\hline \multicolumn{6}{|l|}{$\begin{array}{l}\text { Materiales } \\
\text { Indirectos }\end{array}$} \\
\hline Servicios Básicos & Cantidad & $\begin{array}{c}\text { Unidad } \\
\text { de } \\
\text { medida }\end{array}$ & $\begin{array}{l}\text { Costo unitario } \\
\text { del servicio }\end{array}$ & $\begin{array}{l}\text { Costo total } \\
\text { por Has. }\end{array}$ & Inductor del costo \\
\hline Energía Eléctrica & 1 & & 29.41 & 29.41 & Asignación por lotes \\
\hline Salarios & 5 & & 6.25 & 31.25 & Asignación por lotes \\
\hline Internet & 1 & & 3.52 & 3.52 & Asignación por lotes \\
\hline Telefonía & 1 & & 2.94 & 2.94 & Asignación por lotes \\
\hline \multicolumn{3}{|c|}{ Total de servicios básicos } & 40.87 & \multicolumn{2}{|l|}{67.12} \\
\hline $\begin{array}{l}\text { Gastos por } \\
\text { depreciaciones }\end{array}$ & Cantidad & $\begin{array}{c}\text { Unidad } \\
\text { de } \\
\text { medida }\end{array}$ & $\begin{array}{l}\text { Costo unitario } \\
\text { del servicio }\end{array}$ & $\begin{array}{l}\text { Costo total } \\
\text { por Has. }\end{array}$ & Inductor del costo \\
\hline Maquinaria & 3 & & 294.11 & 882.33 & Asignación por lotes \\
\hline Vehículos & 2 & & 211.76 & 423.52 & Asignación por lotes \\
\hline Caminos & 1 & & 205.88 & 205.88 & Asignación por lotes \\
\hline Empacadora & 1 & & 88.22 & 88.22 & Asignación por lotes \\
\hline \multicolumn{4}{|c|}{ Total de gastos depreciaciones } & $1,599.95$ & \\
\hline
\end{tabular}




\section{Sistema de costos en base actividades}

Tabla 10. Clasificación de costos en base actividades ABC

\begin{tabular}{|c|c|c|c|c|c|c|c|}
\hline Categoría & Código & Descripción & $\begin{array}{c}\text { Precio } \\
\text { Unitario }\end{array}$ & $\begin{array}{l}\text { Cantidad } \\
\text { por } \\
\text { Hectárea }\end{array}$ & $\begin{array}{l}\text { Total por } \\
\text { Hectárea }\end{array}$ & $\begin{array}{c}\% \text { del } \\
\text { valor de } \\
\text { consumo }\end{array}$ & $\begin{array}{c}\% \\
\text { Acumulado }\end{array}$ \\
\hline \multirow{20}{*}{ A } & 1 & Colines & 0,77 & $6.500,00$ & $5.005,00$ & 52,43 & 52,43 \\
\hline & 2 & Nitrogreen & 0,00048 & $75.000,00$ & 35,66 & 0,37 & 52,80 \\
\hline & 3 & Sulfati de Potasio & 0,00 & $25.000,00$ & 16,58 & 0,17 & 52,98 \\
\hline & 4 & Muriato de Potasio & 0,00 & $50.000,00$ & 0,40 & 0,00 & 52,98 \\
\hline & 5 & Kieserete & 0,00 & $25.000,00$ & 6,87 & 0,07 & 53,05 \\
\hline & 6 & Sulfato de Zinc & 0,00 & $15.000,00$ & 21,55 & 0,23 & 53,28 \\
\hline & 7 & Naturcomplet G & 0,00 & $12.500,00$ & 19,13 & 0,20 & 53,48 \\
\hline & 8 & Bazemax & 0,00 & $150.000,00$ & 53,88 & 0,56 & 54,04 \\
\hline & 9 & Echo & 7,55 & 8,75 & 66,06 & 0,69 & 54,74 \\
\hline & 10 & Coadyuvante $\mathrm{x}-50$ & 22,22 & 1,00 & 22,22 & 0,23 & 54,97 \\
\hline & 11 & Servicio de fumigación & 10,90 & 5,00 & 54,50 & 0,57 & 55,54 \\
\hline & 12 & Emthane & 6,90 & 7,50 & 51,75 & 0,54 & 56,08 \\
\hline & 13 & Coadyuvante $\mathrm{x}-50$ & 22,22 & 1,00 & 22,22 & 0,23 & 56,32 \\
\hline & 14 & Servicio de fumigación & 10,90 & 5,00 & 54,50 & 0,57 & 56,89 \\
\hline & 15 & Siganex & 22,25 & 2,50 & 55,63 & 0,58 & 57,47 \\
\hline & 16 & Impulse & 34,90 & 2,00 & 69,80 & 0,73 & 58,20 \\
\hline & 17 & Coadyuvante $\mathrm{x}-50$ & 22,22 & 0,50 & 11,11 & 0,12 & 58,32 \\
\hline & 18 & Aceite agrícola importado & 3,55 & 7,50 & 26,63 & 0,28 & 58,60 \\
\hline & 19 & Servicio de fumigación & 10,50 & 5,00 & 52,50 & 0,55 & 59,15 \\
\hline & 20 & Echo & 7,55 & 8,75 & 66,06 & 0,69 & 59,84 \\
\hline
\end{tabular}




\begin{tabular}{|c|c|c|c|c|c|c|c|}
\hline & Código & Descripción & $\begin{array}{c}\text { Precio } \\
\text { Unitario }\end{array}$ & $\begin{array}{c}\text { Cantidad } \\
\text { por } \\
\text { Hectárea }\end{array}$ & $\begin{array}{l}\text { Total por } \\
\text { Hectárea }\end{array}$ & $\begin{array}{c}\% \text { del } \\
\text { valor de } \\
\text { consumo }\end{array}$ & $\begin{array}{c}\% \\
\text { Acumulado }\end{array}$ \\
\hline & 21 & Coadyuvante $\mathrm{x}-50$ & 22,22 & 1,00 & 22,22 & 0,23 & 60,07 \\
\hline & 22 & Servicio de fumigación & 10,90 & 5,00 & 54,50 & 0,57 & 60,64 \\
\hline \multirow{16}{*}{ B } & 23 & Mantenimiento & 240,00 & 1,14 & 273,60 & 2,87 & 63,51 \\
\hline & 24 & Enfundadores & 240,00 & 1,14 & 273,60 & 2,87 & 66,37 \\
\hline & 25 & Mayordomo & 240,00 & 1,21 & 290,40 & 3,04 & 69,42 \\
\hline & 26 & Deshije & 240,00 & 1,14 & 273,60 & 2,87 & 72,28 \\
\hline & 27 & Siembra & 240,00 & 1,14 & 273,60 & 2,87 & 75,15 \\
\hline & 28 & Bodeguero & 240,00 & 1,14 & 273,60 & 2,87 & 78,01 \\
\hline & 29 & Preparación del suelo & 36,00 & 1,15 & 37,15 & 0,39 & 78,40 \\
\hline & 30 & Siembra & 40,00 & 1,15 & 41,15 & 0,43 & 78,83 \\
\hline & 31 & Arado & 40,00 & 1,15 & 41,15 & 0,43 & 79,27 \\
\hline & 32 & Cuadrilla & 48,00 & 1,00 & 49,00 & 0,51 & 79,78 \\
\hline & 33 & Cuadrilla & 48,00 & 1,00 & 49,00 & 0,51 & 80,29 \\
\hline & 34 & Cuadrilla & 48,00 & 1,00 & 49,00 & 0,51 & 80,80 \\
\hline & 35 & Embarque & 48,00 & 1,00 & 49,00 & 0,51 & 81,32 \\
\hline & 36 & Embarque & 48,00 & 1,00 & 49,00 & 0,51 & 81,83 \\
\hline & 37 & Embarque & 48,00 & 1,00 & 49,00 & 0,51 & 82,34 \\
\hline & 38 & Cinta & 4,00 & 2,79 & 6,79 & 0,07 & 82,42 \\
\hline \multirow{7}{*}{$\mathrm{C}$} & 39 & Pañuelos & 600,00 & 0,05 & 600,05 & 6,29 & 88,70 \\
\hline & 40 & Fundas & 300,00 & 0,08 & 300,08 & 3,14 & 91,85 \\
\hline & 41 & Daypas & 432,00 & 0,06 & 432,06 & 4,53 & 96,37 \\
\hline & 42 & Gomas & 1,40 & 21,68 & 23,08 & 0,24 & 96,61 \\
\hline & 43 & Cloro & 1,36 & 1,21 & 2,57 & 0,03 & 96,64 \\
\hline & 44 & Removedor & 1,00 & 1,20 & 2,20 & 0,02 & 96,66 \\
\hline & 45 & Piola & 1,00 & 1,70 & 2,70 & 0,03 & 96,69 \\
\hline
\end{tabular}


ISSN: 2602-8506

www.visionariodigital.org

Vol. 3, N².1., p. 420-444, junio, 2019

\begin{tabular}{|c|l|l|c|c|c|c|c|}
\hline 46 & Lustre & 2,00 & 0,30 & 2,30 & 0,02 & 96,72 \\
\cline { 2 - 7 } & Código & Descripción & $\begin{array}{c}\text { Precio } \\
\text { Unitario }\end{array}$ & $\begin{array}{c}\text { Cantidad } \\
\text { por } \\
\text { Hectárea }\end{array}$ & $\begin{array}{c}\text { Total por } \\
\text { Hectárea }\end{array}$ & $\begin{array}{c}\text { \% del } \\
\text { valor de } \\
\text { consumo }\end{array}$ & $\begin{array}{c}\text { \% } \\
\text { Acumulado }\end{array}$ \\
\hline 47 & Protectores & 3,00 & 0,04 & 3,04 & 0,03 & 96,75 \\
\hline 48 & Cartones & 231,00 & 0,47 & 231,47 & 2,42 & 99,17 \\
\hline 49 & Etiqueta & 1,00 & 1,90 & 2,90 & 0,03 & 99,20 \\
\hline 50 & Garruchas & 2,00 & 0,06 & 2,06 & 0,02 & 99,22 \\
\hline 51 & Podones & 6,00 & 1,15 & 7,15 & 0,07 & 99,30 \\
\hline 52 & Cucharetas & 5,00 & 1,50 & 6,50 & 0,07 & 99,37 \\
\hline 53 & Cunas & 8,00 & 0,17 & 8,17 & 0,09 & 99,45 \\
\hline 54 & Platos & 3,00 & 0,21 & 3,21 & 0,03 & 99,49 \\
\hline 55 & Rechazadora & 4,00 & 0,15 & 4,15 & 0,04 & 99,53 \\
\hline 56 & Energía Eléctrica & 1,00 & 29,41 & 30,41 & 0,32 & 99,85 \\
\hline 57 & Sueldos Administrativos & 1,00 & 5,00 & 31.25 & 0,06 & 99,91 \\
\hline 58 & Internet & 1,00 & 3,52 & 4,52 & 0,05 & 99,96 \\
\hline
\end{tabular}

Porcentajes de la clasificación de costos ABC

Tabla 11. Porcentajes de costos de acuerdo a la categoría

\begin{tabular}{|c|c|c|}
\hline Categoría & $\begin{array}{l}\text { (\%) de } \\
\text { Categoría }\end{array}$ & $\begin{array}{l}\text { Porcentaje } \\
\text { del costo }\end{array}$ \\
\hline A & 37,29 & 61 \\
\hline B & 25,42 & 22 \\
\hline C & 37,29 & 18 \\
\hline
\end{tabular}

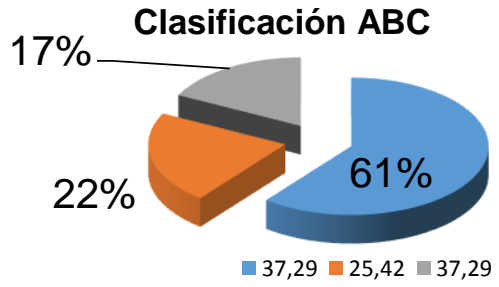


Tabla 12. Porcentajes de acuerdo a los ítems

\begin{tabular}{|c|c|}
\hline Categoría & Cantidad de ítems \\
\hline $\mathrm{A}(37,29)$ & 22 \\
\hline $\mathrm{B}(25,42)$ & 15 \\
\hline $\mathrm{C}(37,29)$ & 22 \\
\hline TOTAL & $\mathbf{5 9}$ \\
\hline
\end{tabular}

\section{Pocertanje de ítems de la clasificación $A B C$}

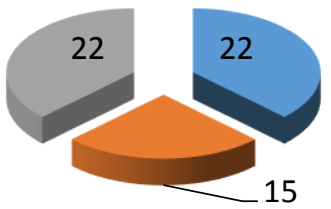

Tabla 13. Representación monetaria de los costos según su categoría

\begin{tabular}{|c|c|}
\hline Categoría & $\begin{array}{c}\text { Costo total por } \\
\text { Hectárea (dólares) }\end{array}$ \\
\hline A & $5.788,75$ \\
\hline B & 2071,85 \\
\hline C & 1703.81 \\
\hline TOTAL & $\mathbf{9 . 5 6 4 . 4 1}$ \\
\hline
\end{tabular}

Clasificacion ABC por costos

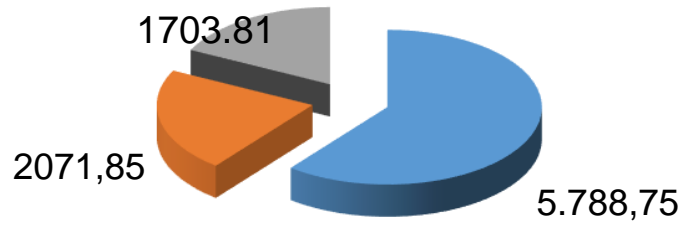

Análisis: Luego de realizar una clasificación de los costos por actividades que incurren en la elaboración de una caja de banano convencional 22xu se encuentran encasillados de acuerdo a sus categorías; categoría A: representa un $61 \%$ esta categoría hace referencia a la preparación del suelo y siembra, categoría B: representa el $22 \%$ en cuanto esta categoría simboliza la actividad de mantenimiento y crecimiento de los costos totales y la categoría C; representa a la actividad de cosecha y embarque con el 17\% del total de los costos, los cuales se clasificaron de acuerdo a los niveles de importancia en cuanto a los inductores del costo que fueron tomados por las ordenes de producción que se realiza en la bananera.

\section{Costos según el sistema en base actividades ABC}

Tabla 14 Costos caja de banano convencional 22xu

\begin{tabular}{l|r}
\hline Categorías & Costo total \\
\hline Costo por Hectárea A & $5.788,75$ \\
Costo por Hectárea B & 2071,85 \\
Costo por Hectárea C & 1703,81 \\
\hline Total ABC & 9.564 .41 \\
\hline Cajas de banano 22xu en 5 Has & $2.000,00$ \\
\hline & \\
Costo de caja unitario de caja de banano & $\mathbf{4 , 7 8}$ \\
\hline
\end{tabular}




\section{Conclusiones.}

$\checkmark$ El costeo en base a actividades permite conocer el valor real por cada actividad que realiza la empresa, es un sistema completo que no desplaza los costos tradicionales, pero los complementa dando facilidad a la identificación de los elementos del costo en las respectivas actividades determinadas mediante este sistema.

$\checkmark$ Se pudo determinar el costo por unidades de la caja de banano convencional 22xu de manera sencilla analizando las actividades que se consideran de mayor importancia dentro de la bananera, además aplicando este sistema de costeo se obtuvo información que la bananera no consideraba para el cálculo dando como resultado una disminución en los costos al momento de emplear costos en base a actividades.

$\checkmark$ Con la aplicación y determinación de los costos en base a las actividades se podrá analizar los resultados que se obtuvieron en esta investigación dando información confiable a los altos directivos de la bananera facilitando la toma decisiones económicas que favorezcan a los intereses de la empresa.

\section{Referencias bibliográficas.}

Baldini, R. (1 de Junio de 2008). Los modelos de costeo y la gestión empresarial -Primera parte. Redalyc, 73-89. Recuperado el 22 de 03 de 2018, de :http://ucsj.redalyc.org/articulo.oa?id=87702007

Barraza, B. (2013). Implicancias del Método de costeo ABC. Quipukamayoc revista de la facultad de ciencias contables.

Cano, A. (2013). Contabilidad Gerencial y Presupuestaria, aplicada a ciencias económicas, administrativas y contables. Bogotá: Ediciones de la U.

Cuevas, V. (2010). Contabilidad de costos enfoque gerencial y de gestión. Bogotá: Pearson.

García, J. (2010). Contabilidad de costos 3era Edición. México: Mc Graw Hill.

Golpe, M. (2017). La gestión estratégica de costos: ABC, ABM y la necesidad de otras clasificaciones de costos para toma de decisiones. Adinet.

Heredia, D. (2008). Metodología de costeo basado en las actividades. Económicas CUC, 89. Recuperado el 3 de 06 de 2019

Horngren, C., Datar, S., \& Rajan, M. (2012). Contabilidad de costos un enfoque gerencial. México: PEARSON.

Lambreton, V., \& Garza, G. (2016). Costos para la toma de decisiones. México: Pearson Educación de México,S.A. de CV. 
Laporta, R. (2016). Costos y gestión empresarial. Bogotá: Ecoe.

Ministerio de Comercio Exterior. (2017). Informe sector bananero ecuatoriano. Quito. Recuperado el 8 de Abril de 2019, de https://www.comercioexterior.gob.ec/wpcontent/uploads/2017/12/Informe-sector-bananero-espa\%C3\%B1ol-04dic17.pdf

Morillo, M. (5 de Enero-junio de 2005). Diseño de sistemas de costeo:. Redalyc, 6. Recuperado el 22 de 03 de 2018, de http://www.redalyc.org/articulo.oa?id=25700507

Ortega, M. (2016). Identificación de activiades para un costeo ABC. Identificación de activiades para un costeo $A B C$. Chile.

Ortíz, A., \& Rivero, G. (2006). Estructuración de costos;conceptos y metodologías. Root Change, 1-28. Recuperado el 9 de Mayo de 2018

Pérez, G. (1996). Metodología de la investigación educacional.

Plaza, E. (2013). Propuesta de costeo mediante ABC.

Polimeni, Fabriozzi, Kole, \& Adelberg. (1997). Contablidad de costos. Bogotá: Lito Camargo Ltda.

Rabalan, A. (2013). Métodos de decisión empresarial. Madrid: Delta publicaciones.

Ramirez, C., Garcia, M., \& Pantoja, C. (2010). Fundamentos y técnicas de costos. Universidad libre, sede Cartagena.

Ramirez, D. (2008). Contabilidad administrativa. México: Mc graw hill.

Torres, A. (2006). Contabilidad de costos. México: Interamericana Editores S.A.

Universidad Eafit . (Octubre de 2007). La importancia de conocer y saber clasificar los costos para una buena toma de decisiones. La importancia de conocer y saber clasificar los costos, Boletin 11, 1-3. Medellin, Colombia: Universidad Eafit.

Vanden, G. (2007). Asignación de costos objetos de costos directos costos indirectos costos variables y costos fijos. Obtenido de https://www.academia.edu/14474193/

Vergíu, J. (2013). La cadena de valor como herramienta de gestión para una empresa de servicios. Revista de la Facultad de Ingeniería Industrial , 18.

Wayne, L., \& Ledesma, J. (2015). Contabilidad para no contadores. España: Piramide.

Zapata, P. (2005). Contabilidad General. Bogotá: Mc Graw Hill.

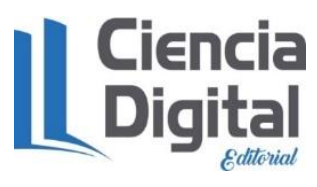




\section{PARA CITAR EL ARTÍCULO INDEXADO.}

Marfetán San Martín, M., Narváez Zurita, C., \& Erazo Álvarez, J. (2019). Pautas para la determinación efectiva del costeo basado en actividades en la industria bananera. Visionario Digital, 3(2.1.), 420-444.

https://doi.org/10.33262/visionariodigital.v3i2.1.583

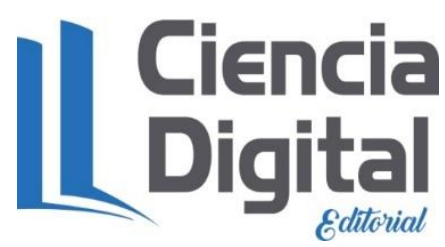

El artículo que se publica es de exclusiva responsabilidad de los autores y no necesariamente reflejan el pensamiento de la Revista Ciencia Digital.

El artículo queda en propiedad de la revista y, por tanto, su publicación parcial y/o total en otro medio tiene que ser autorizado por el director de la Revista Ciencia Digital.
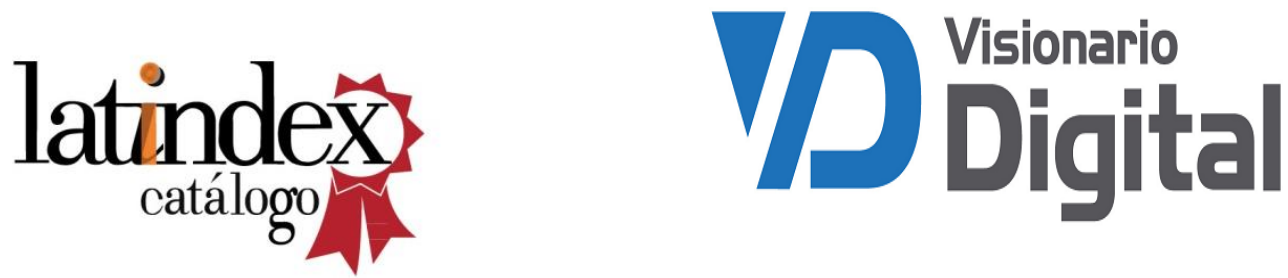